\title{
Erratum to: Quality control in geochemistry from a comparison of four central tendency and five dispersion estimators and example of a geochemical reference material
}

\author{
Surendra P. Verma ${ }^{1}$ • Lorena Díaz-González ${ }^{2}$. Javier A. Pérez-Garza ${ }^{3}$. \\ Mauricio Rosales-Rivera ${ }^{4}$
}

Published online: 6 January 2017

(C) Saudi Society for Geosciences 2017

Erratum to: Arab J Geosci (2016) 9:740

DOI 10.1007/s12517-016-2764-4

A few minor but serious errors occurred in the published article. Please note that throughout the text the symbol $\hat{\sigma}_{n}$ was erroneously replaced by $\sigma \mathrm{n}$. Also note at some places, the standard deviation symbol should be $s$ (and not $S$ ).

More importantly, the headings of Table 2 were misplaced. The correct headings should read as follows:

The online version of the original article can be found under at http://dx. doi.org/10.1007/s12517-016-2764-4.

Surendra P. Verma

spv@ier.unam.mx

1 Instituto de Energías Renovables, Universidad Nacional Autónoma de México, Priv. Xochicalco s/no., Col. Centro, Apdo. 34,

Temixco 62580, Mexico

2 Departamento de Computación, Centro de Investigación en Ciencias, Instituto de Investigación en Ciencias Básicas y Aplicadas,

Universidad Autónoma de Estado de Morelos, Av. Universidad 1001, Chamilpa, Cuernavaca, Mor. 62209, Mexico

3 Licenciatura en Ciencias, Instituto de Investigación en Ciencias Básicas y Aplicadas, Universidad Autónoma de Estado de Morelos, Av. Universidad 1001, Chamilpa, Cuernavaca, Mor. 62209, Mexico

4 Doctorado en Ciencias, Instituto de Investigación en Ciencias Básicas y Aplicadas, Universidad Autónoma de Estado de Morelos, Av. Universidad 1001, Chamilpa, Cuernavaca, Mor. 62209, Mexico 
Table 2 Finite sample correction factors* for several dispersion estimators

\begin{tabular}{|c|c|c|c|c|c|c|c|c|c|c|c|}
\hline \multirow[t]{2}{*}{$n$} & \multicolumn{2}{|l|}{$s$} & \multicolumn{4}{|l|}{$M A D$} & \multicolumn{2}{|l|}{$S_{n}$} & \multicolumn{2}{|l|}{$Q_{n}$} & \multirow{2}{*}{$\begin{array}{l}\widehat{\sigma}_{n} \\
\text { this work }\end{array}$} \\
\hline & Bolch (1968) & this work & CR92** & Williams (2011) & Hayes (2014) & this work & CR92** & this work & CR92** & this work & \\
\hline 5 & 1.06385 & 1.0640 & 1.788 & 1.805 & 1.8038 & 1.8055 & 1.611 & 1.6095 & 1.875 & 1.3656 & 1.3762 \\
\hline 6 & 1.05094 & 1.0513 & 1.779 & 1.763 & 1.7637 & 1.7643 & 1.184 & 1.3834 & 1.358 & 1.3592 & 1.2855 \\
\hline 7 & 1.04235 & 1.0426 & 1.690 & 1.688 & 1.6872 & 1.6877 & 1.429 & 1.4299 & 1.904 & 1.6146 & 1.2587 \\
\hline 8 & 1.03624 & 1.0365 & 1.674 & 1.671 & 1.6714 & 1.6716 & 1.199 & 1.3371 & 1.486 & 1.4874 & 1.2167 \\
\hline 9 & 1.03166 & 1.0319 & 1.641 & 1.633 & 1.6327 & 1.6336 & 1.349 & 1.3501 & 1.937 & 1.6018 & 1.2019 \\
\hline 10 & 1.02811 & 1.0284 & 1.612 & 1.628 & 1.6247 & 1.6252 & 1.193 & 1.3076 & 1.610 & 1.5988 & 1.1781 \\
\hline 11 & 1.02527 & 1.0255 & 1.599 & 1.612 & 1.6013 & 1.6018 & 1.299 & 1.3075 & 1.971 & 1.7323 & 1.1685 \\
\hline 12 & 1.02296 & 1.0232 & 1.589 & 1.599 & 1.5962 & 1.5969 & 1.193 & 1.2862 & 1.688 & 1.6814 & 1.1529 \\
\hline 13 & 1.02103 & 1.0213 & 1.580 & 1.589 & 1.5805 & 1.5810 & 1.281 & 1.2817 & 2.006 & 1.7425 & 1.1464 \\
\hline 14 & 1.01940 & 1.0197 & 1.572 & 1.580 & 1.5773 & 1.5777 & 1.193 & 1.2703 & 1.748 & 1.7434 & 1.1355 \\
\hline 15 & 1.01800 & 1.0184 & 1.566 & 1.573 & 1.5662 & 1.5665 & 1.269 & 1.2647 & 2.032 & 1.8223 & 1.1310 \\
\hline 16 & 1.01679 & 1.0171 & 1.561 & 1.567 & 1.5637 & 1.5640 & 1.193 & 1.2585 & 1.795 & 1.7931 & 1.1229 \\
\hline 17 & 1.01574 & 1.0161 & 1.556 & 1.561 & 1.5555 & 1.5561 & 1.259 & 1.2531 & 2.053 & 1.8325 & 1.1194 \\
\hline 18 & 1.01481 & 1.0152 & 1.552 & 1.556 & 1.5535 & 1.5542 & 1.193 & 1.2494 & 1.835 & 1.8339 & 1.1132 \\
\hline 19 & 1.01398 & 1.0143 & 1.548 & 1.552 & 1.5470 & 1.5479 & 1.252 & 1.2444 & 2.069 & 1.8859 & 1.1104 \\
\hline 20 & 1.01324 & 1.0135 & 1.544 & 1.548 & 1.5458 & 1.5465 & 1.193 & 1.2424 & 1.867 & 1.8671 & 1.1056 \\
\hline 21 & 1.01257 & 1.0129 & 1.541 & 1.545 & 1.5406 & 1.5409 & 1.246 & 1.2377 & 2.083 & 1.8941 & 1.1032 \\
\hline 22 & 1.01197 & 1.0122 & 1.539 & 1.542 & 1.5394 & 1.5400 & 1.193 & 1.2366 & 1.895 & 1.8952 & 1.0992 \\
\hline 23 & 1.01142 & 1.0117 & 1.536 & 1.539 & 1.5352 & 1.5358 & 1.241 & 1.2326 & 2.094 & 1.9319 & 1.0973 \\
\hline 24 & 1.01093 & 1.0111 & 1.534 & 1.537 & 1.5342 & 1.5344 & 1.193 & 1.2318 & 1.918 & 1.9188 & 1.0939 \\
\hline 25 & 1.01047 & 1.0106 & 1.532 & 1.534 & 1.5307 & 1.5310 & 1.237 & 1.2281 & 2.104 & 1.9384 & 1.0924 \\
\hline 26 & 1.01005 & 1.0102 & 1.530 & 1.532 & 1.5300 & 1.5304 & 1.193 & 1.2281 & 1.939 & 1.9395 & 1.0896 \\
\hline 27 & 1.00965 & 1.0098 & 1.528 & 1.530 & 1.5270 & 1.5273 & 1.234 & 1.2249 & 2.112 & 1.9670 & 1.0882 \\
\hline 28 & 1.00931 & 1.0094 & 1.526 & 1.528 & 1.5260 & 1.5264 & 1.193 & 1.2249 & 1.956 & 1.9574 & 1.0858 \\
\hline 29 & 1.00897 & 1.0090 & 1.525 & 1.527 & 1.5239 & 1.5240 & 1.231 & 1.2220 & 2.120 & 1.9723 & 1.0846 \\
\hline 30 & 1.00866 & 1.0087 & 1.523 & 1.525 & 1.5230 & 1.5232 & 1.193 & 1.2222 & 1.972 & 1.9731 & 1.0826 \\
\hline 31 & 1.00836 & 1.0085 & 1.522 & 1.524 & 1.5209 & 1.5212 & 1.228 & 1.2197 & 2.126 & 1.9946 & 1.0817 \\
\hline 32 & 1.00810 & 1.0082 & 1.521 & 1.522 & 1.5205 & 1.5208 & 1.193 & 1.2200 & 1.986 & 1.9871 & 1.0799 \\
\hline 33 & 1.00784 & 1.0079 & 1.519 & 1.521 & 1.5186 & 1.5187 & 1.226 & 1.2177 & 2.131 & 1.9990 & 1.0790 \\
\hline 34 & 1.00760 & 1.0077 & 1.518 & 1.520 & 1.5181 & 1.5184 & 1.193 & 1.2180 & 1.999 & 1.9996 & 1.0775 \\
\hline 35 & 1.00738 & 1.0074 & 1.517 & 1.519 & 1.5163 & 1.5165 & 1.224 & 1.2159 & 2.136 & 2.0167 & 1.0767 \\
\hline 36 & 1.00717 & 1.0072 & 1.516 & 1.518 & 1.5161 & 1.5163 & 1.193 & 1.2164 & 2.010 & 2.0109 & 1.0753 \\
\hline 37 & 1.00697 & 1.0070 & 1.515 & 1.517 & 1.5147 & 1.5146 & 1.222 & 1.2145 & 2.141 & 2.0205 & 1.0746 \\
\hline 38 & 1.00678 & 1.0068 & 1.514 & 1.516 & 1.5142 & 1.5144 & 1.193 & 1.2150 & 2.020 & 2.0210 & 1.0734 \\
\hline 39 & 1.00660 & 1.0067 & 1.514 & 1.515 & 1.5129 & 1.5131 & 1.221 & 1.2132 & 2.145 & 2.0350 & 1.0728 \\
\hline 40 & 1.00643 & 1.0066 & 1.513 & 1.514 & 1.5124 & 1.5128 & 1.193 & 1.2137 & 2.029 & 2.0303 & 1.0717 \\
\hline 41 & 1.00627 & 1.0064 & 1.512 & 1.513 & 1.5110 & 1.5114 & 1.219 & 1.2122 & 2.149 & 2.0381 & 1.0712 \\
\hline 42 & 1.00612 & 1.0063 & 1.511 & 1.513 & 1.5110 & 1.5112 & 1.193 & 1.2124 & 2.038 & 2.0387 & 1.0702 \\
\hline 43 & 1.00597 & 1.0061 & 1.511 & 1.512 & 1.5099 & 1.5101 & 1.218 & 1.2111 & 2.152 & 2.0505 & 1.0697 \\
\hline 44 & 1.00583 & 1.0060 & 1.510 & 1.511 & 1.5097 & 1.5099 & 1.193 & 1.2115 & 2.045 & 2.0465 & 1.0688 \\
\hline 45 & 1.00570 & 1.0059 & 1.509 & 1.510 & 1.5088 & 1.5088 & 1.217 & 1.2102 & 2.155 & 2.0532 & 1.0683 \\
\hline 46 & 1.00557 & 1.0057 & 1.509 & 1.510 & 1.5083 & 1.5085 & 1.193 & 1.2106 & 2.052 & 2.0534 & 1.0675 \\
\hline 47 & 1.00545 & 1.0056 & 1.508 & 1.509 & 1.5074 & 1.5076 & 1.216 & 1.2093 & 2.158 & 2.0633 & 1.0671 \\
\hline 48 & 1.00533 & 1.0055 & 1.508 & 1.509 & 1.5072 & 1.5076 & 1.193 & 1.2098 & 2.059 & 2.0600 & 1.0664 \\
\hline 49 & 1.00522 & 1.0054 & 1.507 & 1.508 & 1.5063 & 1.5066 & 1.215 & 1.2086 & 2.160 & 2.0657 & 1.0659 \\
\hline 50 & 1.00511 & 1.0052 & 1.507 & 1.508 & 1.5060 & 1.5062 & 1.193 & 1.2089 & 2.065 & 2.0659 & 1.0652 \\
\hline 51 & --- & 1.0051 & 1.506 & 1.507 & 1.5053 & 1.5057 & 1.214 & 1.2080 & 2.163 & 2.0744 & 1.0649 \\
\hline
\end{tabular}


Table 2 (continued)

\begin{tabular}{|c|c|c|c|c|c|c|c|c|c|c|c|}
\hline \multirow[t]{2}{*}{$n$} & \multicolumn{2}{|l|}{$s$} & \multicolumn{4}{|l|}{$M A D$} & \multicolumn{2}{|l|}{$S_{n}$} & \multicolumn{2}{|l|}{$Q_{n}$} & \multirow{2}{*}{$\begin{array}{l}\widehat{\sigma}_{n} \\
\text { this work }\end{array}$} \\
\hline & Bolch (1968) & this work & CR92** & Williams (2011) & Hayes (2014) & this work & CR92** & this work & CR92** & this work & \\
\hline 52 & --- & 1.0051 & 1.506 & 1.507 & 1.5051 & 1.5053 & 1.193 & 1.2082 & 2.071 & 2.0716 & 1.0642 \\
\hline 53 & --- & 1.0050 & 1.505 & 1.506 & 1.5047 & 1.5048 & 1.213 & 1.2074 & 2.165 & 2.0764 & 1.0639 \\
\hline 54 & --- & 1.0049 & 1.505 & 1.506 & 1.5044 & 1.5047 & 1.193 & 1.2077 & 2.076 & 2.0769 & 1.0634 \\
\hline 55 & --- & 1.0048 & 1.504 & 1.505 & 1.5038 & 1.5041 & 1.212 & 1.2069 & 2.167 & 2.0840 & 1.0630 \\
\hline 56 & --- & 1.0047 & 1.504 & 1.505 & 1.5035 & 1.5038 & 1.193 & 1.2071 & 2.081 & 2.0816 & 1.0625 \\
\hline 57 & --- & 1.0046 & 1.504 & 1.505 & 1.5029 & 1.5032 & 1.212 & 1.2063 & 2.169 & 2.0857 & 1.0622 \\
\hline 58 & --- & 1.0046 & 1.503 & 1.504 & 1.5029 & 1.5031 & 1.193 & 1.2066 & 2.085 & 2.0861 & 1.0617 \\
\hline 59 & --- & 1.0045 & 1.503 & 1.504 & 1.5024 & 1.5025 & 1.211 & 1.2058 & 2.170 & 2.0925 & 1.0614 \\
\hline 60 & 1.00425 & 1.0044 & 1.503 & 1.503 & 1.5024 & 1.5023 & 1.193 & 1.2061 & 2.090 & 2.0904 & 1.0609 \\
\hline 61 & --- & 1.0043 & 1.502 & 1.503 & 1.5015 & 1.5018 & 1.210 & 1.2054 & 2.172 & 2.0940 & 1.0607 \\
\hline 62 & --- & 1.0043 & 1.502 & 1.503 & 1.5015 & 1.5016 & 1.193 & 1.2056 & 2.094 & 2.0942 & 1.0603 \\
\hline 63 & --- & 1.0042 & 1.502 & 1.502 & 1.5011 & 1.5011 & 1.210 & 1.2049 & 2.174 & 2.0999 & 1.0600 \\
\hline 64 & --- & 1.0041 & 1.501 & 1.502 & 1.5008 & 1.5010 & 1.193 & 1.2052 & 2.097 & 2.0980 & 1.0596 \\
\hline 65 & --- & 1.0041 & 1.501 & 1.502 & 1.5004 & 1.5005 & 1.209 & 1.2045 & 2.175 & 2.1013 & 1.0594 \\
\hline 66 & --- & 1.0040 & 1.501 & 1.502 & 1.5002 & 1.5005 & 1.193 & 1.2048 & 2.101 & 2.1015 & 1.0590 \\
\hline 67 & --- & 1.0040 & 1.501 & 1.501 & 1.4999 & 1.5001 & 1.209 & 1.2043 & 2.176 & 2.1066 & 1.0589 \\
\hline 68 & --- & 1.0039 & 1.500 & 1.501 & 1.4997 & 1.5000 & 1.193 & 1.2045 & 2.104 & 2.1048 & 1.0585 \\
\hline 69 & --- & 1.0039 & 1.500 & 1.501 & 1.4995 & 1.4996 & 1.208 & 1.2039 & 2.178 & 2.1078 & 1.0583 \\
\hline 70 & 1.00363 & 1.0038 & 1.500 & 1.500 & 1.4993 & 1.4994 & 1.193 & 1.2042 & 2.107 & 2.1080 & 1.0579 \\
\hline 71 & 1.0037 & 1.499 & 1.500 & 1.4988 & 1.4991 & 1.208 & 1.2036 & 2.179 & 2.1124 & 1.0577 & \\
\hline 72 & --- & 1.0037 & 1.499 & 1.500 & 1.4988 & 1.4990 & 1.193 & 1.2038 & 2.111 & 2.1110 & 1.0574 \\
\hline 73 & --- & 1.0036 & 1.499 & 1.500 & 1.4984 & 1.4985 & 1.207 & 1.2033 & 2.180 & 2.1135 & 1.0572 \\
\hline 74 & --- & 1.0035 & 1.499 & 1.499 & 1.4984 & 1.4985 & 1.193 & 1.2034 & 2.113 & 2.1136 & 1.0569 \\
\hline 75 & --- & 1.0035 & 1.499 & 1.499 & 1.4981 & 1.4981 & 1.207 & 1.2029 & 2.181 & 2.1177 & 1.0567 \\
\hline 76 & --- & 1.0034 & 1.498 & 1.499 & 1.4979 & 1.4980 & 1.193 & 1.2032 & 2.116 & 2.1163 & 1.0564 \\
\hline 77 & --- & 1.0034 & 1.498 & 1.499 & 1.4977 & 1.4976 & 1.207 & 1.2027 & 2.182 & 2.1187 & 1.0563 \\
\hline 78 & --- & 1.0034 & 1.498 & 1.499 & 1.4975 & 1.4976 & 1.193 & 1.2029 & 2.119 & 2.1188 & 1.0560 \\
\hline 79 & --- & 1.0033 & 1.498 & 1.498 & 1.4972 & 1.4973 & 1.206 & 1.2024 & 2.183 & 2.1225 & 1.0559 \\
\hline 80 & 1.00317 & 1.0033 & 1.498 & 1.498 & 1.4970 & 1.4972 & 1.193 & 1.2026 & 2.121 & 2.1213 & 1.0556 \\
\hline 81 & --- & 1.0032 & 1.497 & 1.498 & 1.4968 & 1.4969 & 1.206 & 1.2022 & 2.184 & 2.1234 & 1.0554 \\
\hline 82 & --- & 1.0032 & 1.497 & 1.498 & 1.4968 & 1.4968 & 1.193 & 1.2024 & 2.123 & 2.1236 & 1.0552 \\
\hline 83 & --- & 1.0032 & 1.497 & 1.498 & 1.4963 & 1.4966 & 1.206 & 1.2020 & 2.185 & 2.1269 & 1.0551 \\
\hline 84 & --- & 1.0031 & 1.497 & 1.497 & 1.4963 & 1.4966 & 1.193 & 1.2021 & 2.126 & 2.1259 & 1.0549 \\
\hline 85 & --- & 1.0031 & 1.497 & 1.497 & 1.4961 & 1.4963 & 1.205 & 1.2019 & 2.186 & 2.1278 & 1.0548 \\
\hline 86 & --- & 1.0031 & 1.497 & 1.497 & 1.4961 & 1.4962 & 1.193 & 1.2020 & 2.128 & 2.1280 & 1.0545 \\
\hline 87 & --- & 1.0030 & 1.496 & 1.497 & 1.4959 & 1.4960 & 1.205 & 1.2016 & 2.187 & 2.1310 & 1.0544 \\
\hline 88 & --- & 1.0030 & 1.496 & 1.497 & 1.4959 & 1.4959 & 1.193 & 1.2017 & 2.130 & 2.1299 & 1.0542 \\
\hline 89 & --- & 1.0030 & 1.496 & 1.497 & 1.4954 & 1.4956 & 1.205 & 1.2014 & 2.187 & 2.1317 & 1.0541 \\
\hline 90 & 1.00281 & 1.0029 & 1.496 & 1.496 & 1.4954 & 1.4956 & 1.193 & 1.2015 & 2.132 & 2.1317 & 1.0538 \\
\hline 95 & --- & 1.0027 & 1.495 & 1.496 & 1.4948 & 1.4948 & 1.204 & 1.2008 & 2.190 & 2.1378 & 1.0531 \\
\hline 100 & 1.00253 & 1.0026 & 1.495 & 1.496 & 1.4941 & 1.4943 & 1.193 & 1.2006 & 2.141 & 2.1402 & 1.0524 \\
\hline
\end{tabular}

*Correction factors reported in some papers had to be recalculated to make them consistent with their definition as "factors," i.e., the values should be multiplication factors; CR92** Croux and Rousseeuw (1992) 